\title{
Resting state functional connectivity in relapsing remitting multiple sclerosis with mild disability - a data driven, whole brain multivoxel pattern analysis study
}

\author{
Gowthami Nair ${ }^{1}$, Sruthi S. Nair¹, K. M. Arun², Paul Camacho, ${ }^{3,4,5}$, Elshal Bava1, Priya \\ Ajayaghosh$^{1}$, Ramshekhar N. Menon ${ }^{1}$, Muralidharan Nair ${ }^{1}$, Chandrasekharan Kesavadas², \\ Sheeba Arnold Anteraper ${ }^{6}$
}

${ }^{1}$ Department of Neurology, Sree Chitra Tirunal Institute for Medical Sciences and Technology, Thiruvananthapuram, Kerala, India

${ }^{2}$ Department of Imaging Sciences and Interventional Radiology, Sree Chitra Tirunal Institute for Medical Sciences and Technology, Thiruvananthapuram, Kerala, India

${ }^{3}$ Beckman Institute for Advanced Science and Technology, University of Illinois at UrbanaChampaign, 405 N. Mathews Ave., Urbana, IL 61801

${ }^{4}$ Neuroscience Program, University of Illinois at Urbana-Champaign, 405 N. Mathews Ave. Urbana, IL 61801

${ }^{5}$ Interdisciplinary Health Science Institute, University of Illinois at Urbana-Champaign, 901

West University Avenue, Suite 201, MC-261, Urbana, IL 61801

${ }^{6}$ Carle Foundation Hospital, Urbana, IL

\begin{abstract}
Multivoxel pattern analysis (MVPA) has emerged as a powerful unbiased approach for generating seed regions of interest (ROIs) in resting-state functional connectivity (RSFC) analysis in a data-driven manner. The aim of the present study was to investigate RSFC differences between persons with relapsing-remitting multiple sclerosis (RRMS) and healthy controls (HCs). We performed a whole-brain connectome-wide MVPA in 50 RRMS patients with expanded disability status scale $\leq 4$ and 50 age and gender-matched HCs. Significant group differences were noted in RSFC in 9 clusters distributed in 7 regions; right middle frontal gyrus, frontal medial cortex, left frontal pole, anterior cingulate gyrus, right middle temporal gyrus, left posterior middle temporal gyrus and right lateral occipital cortex. Whole-brain seed-to-voxel RSFC characterization of these clusters as seed ROIs revealed significantly increased RSFC to the posterior brain regions (bilateral superior lateral occipital cortices, right lingual gyrus and left occipital pole) and reduced connectivity in the anterior and medial regions (right paracingulate gyrus, anterior cingulate gyrus, left amygdala and left frontal orbital cortex) in RRMS compared to HCs. The results of this study agree with the previous reports on abnormalities of RSFC in RRMS, the cognitive and clinical implications of which are discussed herein.
\end{abstract}




\section{INTRODUCTION}

Multiple sclerosis (MS) is a chronic inflammatory and progressive degenerative disease of the central nervous system (CNS). The hallmark feature of this disorder is the presence of disseminated lesions or 'plaques' in multiple areas within the brain, optic nerve and spinal cord (Noseworthy et al., 2000). These lesions are pathologically recognised as hubs of demyelination, inflammation, axonal loss and gliosis in the white and grey matter (Compston \& Coles, 2008). The first symptomatic clinical episode of MS is referred to as clinically isolated syndrome (CIS) which can variously evolve into relapsing-remitting multiple sclerosis (RRMS) and secondaryprogressive multiple sclerosis (SPMS) in the majority, while 10-15\% have a progressive course from onset (primary progressive multiple sclerosis: PPMS) (Lublin et al. 2014).

Technological advancements in neuroimaging have enhanced the diagnostic utility of magnetic resonance imaging (MRI) for MS (Fox et al., 2011). Beyond the common application of MRI in delineating structural abnormalities, functional MRI has emerged as a promising tool for understanding the anomalous brain networks in MS (Tahedl et al., 2018). Connectivity studies indicate that the connectivity or relay of information between different brain regions alters as the disease progresses (Tahedl et al., 2018). These alterations to the connectome impair the network functioning efficiency of brain (Liu et al., 2017; Shu et al., 2016). Abnormalities in resting state functional connectivity (RSFC) have been observed in all the subtypes of MS, including the very early stages. Synchronization in the RSFC components of the default mode network (DMN) was found to be higher in CIS compared to RRMS and healthy controls (HCs). The compensatory cortical reorganization in the resting state networks which facilitate this diminishes in the later stages of the disease (Roosendaal et al., 2010). Cross-sectional studies have suggested a correlation for higher disability and poorer walking performance in MS with decreased RSFC in the default mode, visual, cognitive, and cerebellar networks (Bollaert et al., 2018; Rocca et al., 2018).

Connectivity studies in RRMS have shown a notable lack of consistency in their results which is attributable to the highly variable methodologies and the variability of clinical features of MS (Bonavita et al., 2011; Filippi et al., 2013; Sbardella et al., 2015). Using data driven techniques such as independent component analysis (ICA), it was noted that there was a significant reorganization of RSFC in networks involved in motor, sensory and cognitive processes between RRMS patients and HCs (Faivre et al., 2012). Another study noted that the DMN had weaker RSFC in the anterior and central posterior cingulate cortices in RRMS patients, where the cognitively preserved patients had more significant variations than cognitively impaired patients (Bonavita et al., 2011). More recent studies in RRMS have generated similar results with reduced connectivity demonstrated in networks like the default mode, attention and memory networks (Nejad-Davarani et al., 2016).

Analysis of resting state functional MRI data follow a hypothesis-driven or a data-driven methodology. In the hypothesis-driven approach, the investigator predefines the regions of interest (ROIs) or seeds which are usually guided by results of previous studies or other hypotheses. Techniques such as seed-to-voxel, seed-to-seed, ROI-to-ROI, and higher-level graph theory analysis have been utilised to study the underlying network abnormalities in persons with 
MS (Tahedl et al., 2018). This approach has an inherent risk of bias as the selection of the predefined regions is subjective.

The data-driven whole brain approach is more flexible and open without any pre-set notions. This method helps in finding new components and provides different insights into existing problems (Iraji et al., 2016). A widely used approach in MS is ICA. ICA helps to detect underlying patterns in the functional MRI (fMRI) signal without any prior knowledge about the task or shape of the hemodynamic response. It separates components in the data to identify spatially independent brain networks. Each non-noise component represents a functional network over a time course thereby allowing identification of independent signals from a combination of signals (McKeown et al., 1998; Tahedl et al., 2018). Multivoxel pattern analysis (MVPA) is another data-driven approach to study RSFC. MVPA attempts to discover spatial patterns of fMRI activity that correspond to different experimental conditions (Mahmoudi et al., 2012). It identifies distributed response patterns across voxels within a given time course. In this approach, seed-points are extracted from the group for further whole-brain connectivity analysis (Mahmoudi et al. 2012). MVPA has been sparingly used but is a potentially useful tool for studying RSFC in MS, which presents spatially heterogeneous damage and adaptations to neural pathways (Dineen et al. 2009, Sbardella et al. 2015). The objective of the present study was to examine the RSFC variations in RRMS persons with mild physical disability compared to HCs using MVPA technique.

\section{METHODS}

\section{Ethics:}

The study commenced after approval from the Institutional Ethics Committee. All participants provided written informed consent prior to recruitment.

\section{Participants:}

The study was conducted as part of a larger project on cognitive dysfunction and multimodality MRI in RRMS. For this study, we recruited 50 persons with RRMS aged 18 years or above from the Multiple Sclerosis Clinic of the Institute who fulfilled the criteria for RRMS based on the 2010 revisions of the McDonald criteria (Polman et al., 2011) and were rated at or below 4 on the Kurtzke expanded disability status scale (EDSS). Participants were recruited from December 2017 to June 2020. Potential participants were excluded based on diagnosis of progressive forms of MS, disease onset before 12 years of age, recent clinical relapse, any corticosteroid therapy within one month, or significant systemic co-morbidities. At recruitment, a detailed clinical assessment was done to record the disease details, neuropsychological scores, functional scores (FS) and EDSS. Fifty age- and gender-matched HCs were also recruited for the study. 


\section{Data acquisition:}

The MRI acquisition was performed using the 3T MRI (GE Discovery MR750w) with its standard 24 channel head array coils. Structural images were acquired first with T1-weighted imaging: $\mathrm{TR}=12.6 \mathrm{~ms}$, TE $=5.8 \mathrm{~ms}$, time of inversion $=550 \mathrm{~ms}$, flip angle $=12^{\circ}$, slice thickness $=2 \mathrm{~mm}$, volumes $=1$, slices $=156$, imaging matrix $=512 \times 512$, and in-plane resolution $=0.35 \times 0.35 \mathrm{~mm}^{2}$. The resting state functional MRI sequences were acquired next with the participants instructed to keep their eyes open and stay awake. The protocol used was $\mathrm{TR}=3000 \mathrm{~ms}, \mathrm{TE}=30 \mathrm{~ms}$, flip angle $=80^{\circ}$, slice thickness $=3.2 \mathrm{~mm}$, volumes $=150$, imaging matrix $=64 \times 64$, and in-plane resolution $=3.31 \times 3.31 \mathrm{~mm}^{2}$.

\section{Data pre-processing:}

Pre-processing and RSFC analysis was performed using the CONN toolbox (Whitfield-Gabrieli \& Nieto-Castanon, 2012). The DICOM images were converted into the Neuroimaging Informatics Technology Initiative (NIfTI) format for further processing. Pre-processing consisted of removal of the initial five functional MRI (fMRI) volumes, followed by functional realignment and unwarping based on field maps. These images were then corrected for their slice-timing, followed by functional segmentation and structural segmentation of the T1weighted images into grey matter, white matter and cerebrospinal fluid after which they were normalized onto the Montreal Neurological Institute (MNI) template space. This was followed by functional normalization and outlier detection. Finally, the images were smoothed with a full width half maximum (FWHM) Gaussian kernel of $8 \mathrm{~mm}$. Temporal band-pass filtering was performed for a frequency range between 0.008 to $0.09 \mathrm{~Hz}$.

\section{Multivoxel pattern analysis:}

Whole-brain MVPA identified seed ROIs for further characterization. The number of factors for group-MVPA was limited to five with a dimensionality reduction of 64. An F-test was performed to average all five MVPA components simultaneously in a single second-level analysis to identify significant differences in whole-brain connectivity between the two groups. The clusters were identified with the default atlas included within the CONN toolbox which is a combination of two atlases - (i) an atlas of cortical and subcortical areas from the FSL HarvardOxford Atlas and cerebellar areas from the AAL and (ii) an atlas of a few common networks and regions such as the DMN, medial prefrontal cortex, posterior cingulate cortex and the left and right parietal cortices.

Based on the F-test results, a mask was extracted, consisting of clusters surviving at a height threshold of $\mathrm{p}<0.001$ and a cluster-size FDR correction of 0.05 . This mask was then utilised to perform a seed-to-voxel RSFC characterization using a t-test between HCs and RRMS patients. The mask was also segregated to obtain separate ROIs for the MVPA-derived F-test clusters which were nine in total. A one sample t-test was utilised separately for all nine clusters on the HCs and patient groups where each cluster had an FDR height-threshold of $\mathrm{p}<0.001$ and cluster threshold of $\mathrm{p}<0.05$ FDR- corrected. 


\section{RESULTS}

\section{Participants:}

The study had 32 females and 18 males each in the RRMS and HC groups. The mean age for the RRMS group was $32.8( \pm 8.0)$ years and the HC group was $31.96( \pm 7.5)$ years. The clinical characteristics of the participants are described in Table 1.

\section{Group MVPA results:}

After executing a whole-brain connectome-wide MVPA between the two groups, we found nine statically significant clusters distributed over seven regions, namely, anterior cingulate gyrus, frontal medial cortex, right middle frontal gyrus, left frontal pole, right middle temporal gyrus, left posterior middle temporal gyrus and right lateral occipital cortex (Figure 1).

\section{Post-hoc seed-to-voxel analysis of MVPA results:}

The nine regions which were identified by MVPA were extracted as a mask containing voxels of significance. Using this mask as seed region, a post-hoc seed-to-voxel analysis revealed the regions with marked variation in connectivity between RRMS patients and HCs. Figure 2 illustrates the regions identified by using the entire mask as the seed whereas the connectivity patterns of the individual clusters are summarized in table 2.

Cluster one and cluster eight were identified in the right middle frontal gyrus (MFG). Both the clusters showed increased connectivity with bilateral superior lateral occipital cortices (LOC) and bilateral frontal poles (FP) in RRMS patients. With cluster 8 as seed, precuneus showed the largest cluster size with increased connectivity in the RRMS group. A decrease in RSFC in RRMS was noted in bilateral inferior frontal gyrus pars opercularis and anterior supramarginal gyrus. The anterior cingulate cortex (ACC) had increased connectivity in cluster 1, but reduced connectivity in cluster 8 in the RRMS group.

The frontal medial cortex was cluster two and showed increased connectivity to bilateral superior LOC in the RRMS group. The third MVPA cluster involved the left FP which showed increased RSFC with precuneus in the RRMS group. Left middle temporal gyrus (MTG), cerebellum crus 2, right frontal orbital cortex, and left frontal gyrus had decreased functional connectivity in RRMS group.

The MVPA clusters four and five were identified in ACC. Right superior frontal gyrus, bilateral angular gyri, bilateral posterior MTG, right FP, right frontal orbital cortex, right MFG, right posterior supramarginal gyrus, and left anterior supramarginal gyrus showed decreased connectivity in the RRMS group. The left postcentral gyrus, the right superior LOC, cerebellum, and left occipital pole exhibited increased connectivity in the RRMS group.

Cluster six was the right LOC wherein the RRMS group had increased connectivity in the precuneus, bilateral posterior MTG, and right superior frontal gyrus. Cluster seven was in the right MTG. With right MTG as a seed, the RRMS group showed significantly increased 
connectivity in bilateral superior LOC and right inferior frontal gyrus, and decreased connectivity to the right paracingulate gyrus.

The final cluster (cluster 9) was the left posterior MTG, from which the RRMS group had decreased connectivity with the bilateral FP, left paracingulate gyrus and right inferior temporal gyrus. The largest cluster here was the right superior LOC, which had increased RSFC along with left superior parietal lobule and left inferior LOC.

The post-hoc analysis showed an overall pattern of increased connectivity in the posterior brain regions and decreased RSFC in the anteromedial regions for RRMS. A reduction in the RSFC was noted over the right paracingulate gyrus, anterior cingulate gyrus, left amygdala and left frontal orbital cortex and an increased RSFC in the right middle frontal gyrus, bilateral superior occipital cortex, right lingual gyrus and left occipital pole in the RRMS (Figure 2).

\section{DISCUSSION}

The main objective of this study was to determine the differences in the RSFC in persons with RRMS compared to HCs using an unbiased data-driven approach. We identified the following 9 significant clusters of altered connectivity between the groups in 7 regions in group MVPA: (1) anterior cingulate gyrus, (2) frontal medial cortex, (3) right middle frontal gyrus, (4) left frontal pole, (5) right posterior middle temporal gyrus, (6) left posterior middle temporal gyrus, and (7) right superior lateral occipital cortex. These clusters correspond to regions in various networks associated with regulating language, attention, executive functions and visual perception (Euston et al., 2012; Koyama et al., 2017; Nagy et al., 2012; Semendeferi et al., 2001; Stevens et al., 2011; Xu et al., 2015). The general pattern revealed in the post-hoc analysis was that of increased RSFC in posterior brain regions and decreased RSFC in the anteromedial regions in RRMS. These results could reflect the specific cognitive and higher order motor and sensory deficits in MS or the compensatory reorganization of networks congruous to the disease stage and disability.

The ACC had reduced RSFC to bilateral angular gyri and MTG, the former being an important component of DMN (Andrews-Hanna et al., 2014). Increased connectivity of ACC to angular gyri and MTG has been linked with better cognitive performance in MS (Loitfelder et al., 2012). ACC has been implicated in integrating emotional and sensory information (Downar et al., 2000; Menon, 2015). The salience network, which is mainly comprised of the anterior insula and the dorsal ACC, plays a crucial role in identifying relevant stimuli from our immediate surroundings to help guide behaviour (Menon, 2015; Qadir et al., 2018). Functionally, ACC has been linked to decision making, social interactions, emotional responses, and control of motor responses (Lavin et al., 2013; Menon, 2015). A clinical study in RRMS found that changes in the connectivity patterns of the ACC were indicative of the restoration of attention and executive functions in the patients (Parisi et al., 2014). Additionally, cortical thinning of the ACC in MS has been linked to specific lateralized deficits; impaired verbal fluency and figural fluency were associated with left and right ACC thinning respectively (Geisseler et al., 2015). 
Middle frontal gyrus registered a decreased RSFC to insular cortex and anterior cingulate gyrus which are components of the salience network (Menon, 2015) and had increased RSFC to the precuneus which is a part of the DMN (Utevsky et al., 2014). The MFG is thought to influence both the dorsal and ventral attention networks by reorienting and interrupting attention to novel stimulus (Corbetta et al., 2008, Fox et al., 2011). The MFG has also been described as being involved in cognitive functions involving literacy and numeracy (Koyama et al., 2017) and as a core region in attention (Japee et al., 2015). Decreased activation of left MFG was registered along with abnormalities in DMN activation in MS compared to controls using a working memory network recruiting fMRI paradigm (Vacchi et al., 2016). Depressed MS persons had abnormal RSFC in important networks including DMN and salience networks and increased connectivity to right MFG compared to non-depressed MS persons. (Bonavita et al.,2016)

The frontal medial cortex was noted to have significant variation in RSFC, and when placed as a seed region, had predominantly decreased connectivity to the bilateral superior lateral occipital cortex. This region can be divided into the anterior cingulate cortex, supplementary motor area (SMA), the pre-SMA, orbitofrontal cortex, and anterior frontal poles (Amodio \& Frith, 2006). It has been linked to multiple cognitive functions including decision making and action monitoring (Moreira et al., 2016). Indeed, decision making time was correlated with diffusion abnormalities in medial prefrontal, middle frontal and anterior cingulate cortices in MS compared to controls (Mulhert et al. 2014). Aberrant medial frontal activation resulted on administration of tasks with increasing complexity in RRMS suggesting adaptive recruitment of this region to maintain cognitive performance (Bonnet 2010). A similar phenomenon of increased connectivity between precuneus and medial frontal cortex was noted in cognitively preserved pediatric onset MS with increasing FC correlated with decreasing cognitive performance (Akbar et al.,2015).

The FP, also highlighted in the MVPA results, is involved in cognitive functions such as working memory, reward and conflict, risk, and decision making (Peng et al., 2018). This region has prominent connections extending to the thalamus, basal ganglia, and hypothalamus (Peng et al., 2018). In a study in RRMS, cerebral perfusion techniques were utilised to quantify cortical dysfunction (Vitorino et al., 2016). Cognitive impairment in MS was associated with reductions in cerebral blood flow and cerebral blood volume in Brodman's area 10 (corresponding to the frontal polar regions) which have been previously linked with prospective memory and "intentional forgetting”. This study suggested that these regions may have a role in controlling and manipulating memory in RRMS (Vitorino et al., 2016).

Increased RSFC was recorded from left and right lateral occipital cortices (LOC), with the right LOC having significantly increased connectivity to the precuneus in the RRMS group. This region has been linked to areas concerned with high activation for faces (Nagy et al., 2012). An article studying the plasticity of visual system in MS indicated that the LOC has a role in the visual recovery, with adaptive neuroplasticity, where overactivation of this region was associated with better visual outcomes (Gallo et al., 2015). It was also observed that the functional connectivity between the fusiform gyrus and the LOC was reduced in MS patients compared to controls and the connectivity between these two regions was positively associated with implicit emotional recognition (Golde et al., 2020). 
The left and right middle temporal gyrus (MTG) were the other regions with significant variation in connectivity. Imaging studies have indicated the involvement of the MTG in cognitive functions like language, semantic memory processing and multimodal sensory integration, with the posterior MTG mainly linked to language processing (Onitsuka et al., 2004; Xu et al., 2015). Clinically, MS patients diagnosed with seizures have been found to have grey matter lesions in the MTG and reduced neuronal number in histopathology of this region (Nicholas et al., 2016). Since an increase in seizures is hypothesized to stem from neuronal dysfunction or damage, it could be implied that the variation in RSFC was also a result of such damage. However, further studies are needed to verify this postulation.

An increased connectivity to precuneus was noted from three of the group MVPA-derived clusters, namely, right MFG, left FP and right LOC. Previous studies have suggested that this increase in connectivity could represent a compensatory mechanism attempting to limit the extent of functional damage caused due to loss of tissue (Rocca et al., 2010). This could also indicate recruitment of additional brain regions adjacent to region of damage to support processing in the remaining functioning areas (Droby et al., 2016). The superior LOC was highlighted to have increased RSFC in RRMS group in 8 out of the 9 seed regions. In a recent study, the LOC was observed as being a part of the left and right frontoparietal networks; both thought to be involved in higher-order cognitive functioning affected in RRMS (Janssen et al., 2013). The left frontoparietal network has shown associations with language processing, working memory, and attention (Janssen et al., 2013). The right frontoparietal network has been implicated in pain, sensory perception, and cognitive control (Janssen et al., 2013). The observed increased connectivity could contribute to compensatory mechanisms for preserving these cognitive functions for which typical pathways have been damaged by demyelination and neurodegeneration in RRMS.

The primary strength of our study was the use of a data-driven approach based on MVPA which allowed for an unbiased method for comparison of RSFC between persons with RRMS and ageand sex-matched HCs. This is one of the pioneer studies where this technique has been used in MS. Our major limitation was the lack of information on the correlation of neuropsychological test scores and physical disability with RSFC abnormalities within our sample. This data could have provided more directly translatable clinical insight into the RSFC changes in each network with respect to disability in RRMS. Similarly, we did not correlate the network changes with structural imaging studies of cortical thickness or diffusion tensor imaging. The cross-sectional nature of the study precluded hypotheses on causal nature of the observed changes and information on network dysfunction versus compensatory changes in connectivity. The use of a relatively homogeneous group of RRMS with mild disability was considered important as the resting state data in MS tends to show marked variation over the disease course (Sbardella et al. 2015). However, even this group included patients with a notable variability in duration, symptoms, and MRI lesion load. The duration of formal education was not comparable between the RRMS and HC groups and the influence of the same in network connectivity is not wellunderstood. Future studies focussing on structural MRI and clinical correlation with the networks 
affected could help in better understanding of the mechanisms of cognitive dysfunction in RRMS. Long term longitudinal studies are essential to identify the moulding of networks in patients over time and their correlation with the disease course and disability in MS.

We have reported the RSFC abnormalities in patients of RRMS using the relatively novel technique of MVPA. The post-hoc results revealed a general pattern of increased RSFC in the posterior brain regions and reduced connectivity in the anterior regions. Precuneus and lateral occipital cortex were seen to have consistent abnormalities with increased connectivity in the RRMS group compared to the HCs. Previous studies of RSFC in RRMS have shown wide variability in the techniques and outcomes (Bonavita et al., 2011; Filippi et al., 2013; Sbardella et al., 2015). Further research utilising MVPA could provide unbiased imaging biomarkers for monitoring sub-clinical disease progression and intervention response in RRMS. MVPA-based RSFC abnormalities may be useful in understanding the mechanisms that alter the networks in RRMS and their influence on the highly variable clinical presentations observed across patients.

\section{REFERENCES}

1. Akbar, N., Till, C., Sled, J. G., Binns, M. A., Doesburg, S. M., Aubert-Broche, B., Collins, D. L., Araujo, D., Narayanan, S., Arnold, D. L., Lysenko, M., \& Banwell, B. (2016). Altered resting-state functional connectivity in cognitively preserved pediatriconset MS patients and relationship to structural damage and cognitive performance. Multiple sclerosis (Houndmills, Basingstoke, England), 22(6), 792-800.

2. Amodio, D. M., \& Frith, C. D. (2006). Meeting of minds: The medial frontal cortex and social cognition. Nature Reviews. Neuroscience, 7(4), 268-277. https://doi.org/10.1038/nrn1884

3. Andrews-Hanna, J. R., Smallwood, J., \& Spreng, R. N. (2014). The default network and self-generated thought: Component processes, dynamic control, and clinical relevance. Annals of the New York Academy of Sciences, 1316(1), 29-52. https://doi.org/10.1111/nyas.12360

4. Bollaert, R. E., Poe, K., Hubbard, E. A., Motl, R. W., Pilutti, L. A., Johnson, C. L., \& Sutton, B. P. (2018). Associations of functional connectivity and walking performance in multiple sclerosis. Neuropsychologia, 117, 8-12. https://doi.org/10.1016/j.neuropsychologia.2018.05.007

5. Bonavita, S., Gallo, A., Sacco, R., Corte, M. D., Bisecco, A., Docimo, R., Lavorgna, L., Corbo, D., Costanzo, A. D., \& Tortora, F. (2011). Distributed changes in default-mode resting-state connectivity in multiple sclerosis. Multiple Sclerosis Journal, 17(4), 411422.

6. Bonavita, S., Sacco, R., Esposito, S., d'Ambrosio, A., Della Corte, M., Corbo, D., Docimo, R., Gallo, A., Lavorgna, L., Cirillo, M., Bisecco, A., Esposito, F., \& Tedeschi, G. (2017). Default mode network changes in multiple sclerosis: a link between depression and cognitive impairment?. European journal of neurology, 24(1), 27-36. https://doi.org/10.1111/ene.13112 
7. Bonnet, M. C., Allard, M., Dilharreguy, B., Deloire, M., Petry, K. G., \& Brochet, B. (2010). Cognitive compensation failure in multiple sclerosis. Neurology, 75(14), 12411248. https://doi.org/10.1212/WNL.0b013e3181f612e3

8. Compston, A., \& Coles, A. (2008). Multiple sclerosis. The Lancet, 372(9648), 15021517. https://doi.org/10.1016/S0140-6736(08)61620-7

9. Corbetta, M., Patel, G., \& Shulman, G. L. (2008). The Reorienting System of the Human Brain: From Environment to Theory of Mind. Neuron, 58(3), 306-324. https://doi.org/10.1016/j.neuron.2008.04.017

10. Dineen, R. A., Vilisaar, J., Hlinka, J., Bradshaw, C. M., Morgan, P. S., Constantinescu, C. S., \& Auer, D. P. (2009). Disconnection as a mechanism for cognitive dysfunction in multiple sclerosis. Brain: a journal of neurology, 132(Pt 1), 239-249. https://doi.org/10.1093/brain/awn275

11. Downar, J., Crawley, A. P., Mikulis, D. J., \& Davis, K. D. (2000). A multimodal cortical network for the detection of changes in the sensory environment. Nature Neuroscience, 3(3), 277-283. https://doi.org/10.1038/72991

12. Droby, A., Yuen, K. S., Muthuraman, M., Reitz, S.-C., Fleischer, V., Klein, J., Gracien, R.-M., Ziemann, U., Deichmann, R., \& Zipp, F. (2016). Changes in brain functional connectivity patterns are driven by an individual lesion in MS: A resting-state fMRI study. Brain Imaging and Behavior, 10(4), 1117-1126.

13. Euston, D. R., Gruber, A. J., \& McNaughton, B. L. (2012). The Role of Medial Prefrontal Cortex in Memory and Decision Making. Neuron, 76(6), 1057-1070. https://doi.org/10.1016/j.neuron.2012.12.002

14. Faivre, A., Rico, A., Zaaraoui, W., Crespy, L., Reuter, F., Wybrecht, D., Soulier, E., Malikova, I., Confort-Gouny, S., Cozzone, P. J., Pelletier, J., Ranjeva, J.-P., \& Audoin, B. (2012). Assessing brain connectivity at rest is clinically relevant in early multiple sclerosis. Multiple Sclerosis Journal, 18(9), 1251-1258. https://doi.org/10.1177/1352458511435930

15. Filippi, M., Agosta, F., Spinelli, E. G., \& Rocca, M. A. (2013). Imaging resting state brain function in multiple sclerosis. Journal of Neurology, 260(7), 1709-1713.

16. Fox, R. J., Beall, E., Bhattacharyya, P., Chen, J., \& Sakaie, K. (2011). Advanced MRI in Multiple Sclerosis: Current Status and Future Challenges. Neurologic Clinics, 29(2), 357-380. https://doi.org/10.1016/j.ncl.2010.12.011

17. Gallo, A., Bisecco, A., Bonavita, S., \& Tedeschi, G. (2015). Functional Plasticity of the Visual System in Multiple Sclerosis. Frontiers in Neurology, 6. https://doi.org/10.3389/fneur.2015.00079

18. Geisseler, O., Pflugshaupt, T., Bezzola, L., Reuter, K., Weller, D., Schuknecht, B., Brugger, P., \& Linnebank, M. (2015). Cortical thinning in the anterior cingulate cortex predicts multiple sclerosis patients' fluency performance in a lateralised manner. NeuroImage : Clinical, 10, 89-95. https://doi.org/10.1016/j.nicl.2015.11.008

19. Golde, S., Heine, J., Pöttgen, J., Mantwill, M., Lau, S., Wingenfeld, K., Otte, C., Penner, I.-K., Engel, A. K., Heesen, C., Stellmann, J.-P., Dziobek, I., Finke, C., \& Gold, S. M. (2020). Distinct Functional Connectivity Signatures of Impaired Social Cognition in Multiple Sclerosis. Frontiers in Neurology, 11. https://doi.org/10.3389/fneur.2020.00507 
20. Iraji, A., Calhoun, V. D., Wiseman, N. M., Davoodi-Bojd, E., Avanaki, M. R. N., Haacke, E. M., \& Kou, Z. (2016). The connectivity domain: Analyzing resting state fMRI data using feature-based data-driven and model-based methods. NeuroImage, 134, 494507. https://doi.org/10.1016/j.neuroimage.2016.04.006

21. Janssen, A. L., Boster, A., Patterson, B. A., Abduljalil, A., \& Prakash, R. S. (2013). Resting-state functional connectivity in multiple sclerosis: An examination of group differences and individual differences. Neuropsychologia, 51(13), 2918-2929. https://doi.org/10.1016/j.neuropsychologia.2013.08.010

22. Japee, S., Holiday, K., Satyshur, M. D., Mukai, I., \& Ungerleider, L. G. (2015). A role of right middle frontal gyrus in reorienting of attention: A case study. Frontiers in Systems Neuroscience, 9. https://doi.org/10.3389/fnsys.2015.00023

23. Koyama, M. S., O’Connor, D., Shehzad, Z., \& Milham, M. P. (2017). Differential contributions of the middle frontal gyrus functional connectivity to literacy and numeracy. Scientific Reports, 7(1), 17548. https://doi.org/10.1038/s41598-017-17702-6

24. Lavin, C., Melis, C., Mikulan, E. P., Gelormini, C., Huepe, D., \& Ibanez, A. (2013). The anterior cingulate cortex: An integrative hub for human socially-driven interactions. Frontiers in Neuroscience, 7. https://doi.org/10.3389/fnins.2013.00064

25. Liu, Y., Wang, H., Duan, Y., Huang, J., Ren, Z., Ye, J., Dong, H., Shi, F., Li, K., \& Wang, J. (2017). Functional Brain Network Alterations in Clinically Isolated Syndrome and Multiple Sclerosis: A Graph-based Connectome Study. Radiology, 282(2), 534-541. https://doi.org/10.1148/radiol.2016152843

26. Loitfelder, M., Filippi, M., Rocca, M., Valsasina, P., Ropele, S., Jehna, M., Fuchs, S., Schmidt, R., Neuper, C., Fazekas, F., \& Enzinger, C. (2012). Abnormalities of Resting State Functional Connectivity Are Related to Sustained Attention Deficits in MS. PLOS ONE, 7(8), e42862. https://doi.org/10.1371/journal.pone.0042862

27. Lublin, F. D., Reingold, S. C., Cohen, J. A., Cutter, G. R., Sørensen, P. S., Thompson, A. J., Wolinsky, J. S., Balcer, L. J., Banwell, B., Barkhof, F., Bebo, B., Calabresi, P. A., Clanet, M., Comi, G., Fox, R. J., Freedman, M. S., Goodman, A. D., Inglese, M., Kappos, L., ... Polman, C. H. (2014). Defining the clinical course of multiple sclerosis. Neurology, 83(3), 278-286. https://doi.org/10.1212/WNL.0000000000000560

28. Mahmoudi, A., Takerkart, S., Regragui, F., Boussaoud, D., \& Brovelli, A. (2012). Multivoxel Pattern Analysis for fMRI Data: A Review. Computational and Mathematical Methods in Medicine, 2012, e961257. https://doi.org/10.1155/2012/961257

29. McKeown, M. J., Makeig, S., Brown, G. G., Jung, T. P., Kindermann, S. S., Bell, A. J., \& Sejnowski, T. J. (1998). Analysis of fMRI data by blind separation into independent spatial components. Human Brain Mapping, 6(3), 160-188.

30. Menon, V. (2015). Salience Network. In Brain Mapping (pp. 597-611). Elsevier. https://doi.org/10.1016/B978-0-12-397025-1.00052-X

31. Muhlert, N., Sethi, V., Cipolotti, L., Haroon, H., Parker, G. J., Yousry, T., WheelerKingshott, C., Miller, D., Ron, M., \& Chard, D. (2015). The grey matter correlates of impaired decision-making in multiple sclerosis. Journal of neurology, neurosurgery, and psychiatry, 86(5), 530-536. https://doi.org/10.1136/jnnp-2014-308169 
32. Nagy, K., Greenlee, M. W., \& Kovács, G. (2012). The Lateral Occipital Cortex in the Face Perception Network: An Effective Connectivity Study. Frontiers in Psychology, 3. https://doi.org/10.3389/fpsyg.2012.00141

33. Nejad-Davarani, S. P., Chopp, M., Peltier, S., Li, L., Davoodi-Bojd, E., Lu, M., BagherEbadian, H., Budaj, J., Gallagher, D., Ding, Y., Hearshen, D., Jiang, Q., \& Cerghet, M. (2016). Resting state fMRI connectivity analysis as a tool for detection of abnormalities in five different cognitive networks of the brain in Multiple Sclerosis patients. Clinical Case Reports and Reviews, 2(9), 464-471. https://doi.org/10.15761/CCRR.1000S1001

34. Nicholas, R., Magliozzi, R., Campbell, G., Mahad, D., \& Reynolds, R. (2016). Temporal lobe cortical pathology and inhibitory GABA interneuron cell loss are associated with seizures in multiple sclerosis. Multiple Sclerosis (Houndmills, Basingstoke, England), 22(1), 25-35.

35. Noseworthy, J. H., Lucchinetti, C., Rodriguez, M., \& Weinshenker, B. G. (2000). Multiple sclerosis. The New England journal of medicine, 343(13), 938-952.

36. Onitsuka, T., Shenton, M. E., Salisbury, D. F., Dickey, C. C., Kasai, K., Toner, S. K., Frumin, M., Kikinis, R., Jolesz, F. A., \& McCarley, R. W. (2004). Middle and Inferior Temporal Gyrus Gray Matter Volume Abnormalities in Chronic Schizophrenia: An MRI Study. The American Journal of Psychiatry, 161(9), 1603-1611. https://doi.org/10.1176/appi.ajp.161.9.1603

37. Parisi, L., Rocca, M. A., Valsasina, P., Panicari, L., Mattioli, F., \& Filippi, M. (2014). Cognitive rehabilitation correlates with the functional connectivity of the anterior cingulate cortex in patients with multiple sclerosis. Brain Imaging and Behavior, 8(3), 387-393. https://doi.org/10.1007/s11682-012-9160-9

38. Peng, K., Steele, S. C., Becerra, L., \& Borsook, D. (2018). Brodmann Area 10: Collating, Integrating and High Level Processing of Nociception and Pain. Progress in Neurobiology, 161, 1-22. https://doi.org/10.1016/j.pneurobio.2017.11.004

39. Polman, C. H., Reingold, S. C., Banwell, B., Clanet, M., Cohen, J. A., Filippi, M., Fujihara, K., Havrdova, E., Hutchinson, M., Kappos, L., Lublin, F. D., Montalban, X., O'Connor, P., Sandberg-Wollheim, M., Thompson, A. J., Waubant, E., Weinshenker, B., \& Wolinsky, J. S. (2011). Diagnostic criteria for multiple sclerosis: 2010 revisions to the McDonald criteria. Annals of neurology, 69(2), 292-302.

40. Qadir, H., Krimmel, S. R., Mu, C., Poulopoulos, A., Seminowicz, D. A., \& Mathur, B. N. (2018). Structural Connectivity of the Anterior Cingulate Cortex, Claustrum, and the Anterior Insula of the Mouse. Frontiers in Neuroanatomy, 12. https://doi.org/10.3389/fnana.2018.00100

41. Rocca, M. A., Valsasina, P., Absinta, M., Riccitelli, G., Rodegher, M. E., Misci, P., Rossi, P., Falini, A., Comi, G., \& Filippi, M. (2010). Default-mode network dysfunction and cognitive impairment in progressive MS. Neurology, 74(16), 1252-1259. https://doi.org/10.1212/WNL.0b013e3181d9ed91

42. Rocca, M. A., Valsasina, P., Leavitt, V. M., Rodegher, M., Radaelli, M., Riccitelli, G. C., Martinelli, V., Martinelli-Boneschi, F., Falini, A., Comi, G., \& Filippi, M. (2018). Functional network connectivity abnormalities in multiple sclerosis: Correlations with 
disability and cognitive impairment. Multiple Sclerosis Journal, 24(4), 459-471. https://doi.org/10.1177/1352458517699875

43. Roosendaal, S. D., Schoonheim, M. M., Hulst, H. E., Sanz-Arigita, E. J., Smith, S. M., Geurts, J. J. G., \& Barkhof, F. (2010). Resting state networks change in clinically isolated syndrome. Brain, 133(6), 1612-1621. https://doi.org/10.1093/brain/awq058

44. Sbardella, E., Petsas, N., Tona, F., \& Pantano, P. (2015). Resting-State fMRI in MS: General Concepts and Brief Overview of Its Application. BioMed Research International, 2015, 212693. https://doi.org/10.1155/2015/212693

45. Semendeferi, K., Armstrong, E., Schleicher, A., Zilles, K., \& Hoesen, G. W. V. (2001). Prefrontal cortex in humans and apes: A comparative study of area 10. American Journal of Physical Anthropology, 114(3), 224-241. https://doi.org/10.1002/10968644(200103)114:3<224::AID-AJPA1022>3.0.CO;2-I

46. Shu, N., Duan, Y., Xia, M., Schoonheim, M. M., Huang, J., Ren, Z., Sun, Z., Ye, J., Dong, H., Shi, F.-D., Barkhof, F., Li, K., \& Liu, Y. (2016). Disrupted topological organization of structural and functional brain connectomes in clinically isolated syndrome and multiple sclerosis. Scientific Reports, 6, 29383. https://doi.org/10.1038/srep29383

47. Stevens, F. L., Hurley, R. A., Taber, K. H., Hurley, R. A., Hayman, L. A., \& Taber, K. H. (2011). Anterior Cingulate Cortex: Unique Role in Cognition and Emotion. The Journal of Neuropsychiatry and Clinical Neurosciences, 23(2), 121-125. https://doi.org/10.1176/jnp.23.2.jnp121

48. Tahedl, M., Levine, S. M., Greenlee, M. W., Weissert, R., \& Schwarzbach, J. V. (2018). Functional Connectivity in Multiple Sclerosis: Recent Findings and Future Directions. Frontiers in Neurology, 9. https://doi.org/10.3389/fneur.2018.00828

49. Utevsky, A. V., Smith, D. V., \& Huettel, S. A. (2014). Precuneus Is a Functional Core of the Default-Mode Network. Journal of Neuroscience, 34(3), 932-940. https://doi.org/10.1523/JNEUROSCI.4227-13.2014

50. Vacchi, L., Rocca, M. A., Meani, A., Rodegher, M., Martinelli, V., Comi, G., Falini, A., \& Filippi, M. (2017). Working memory network dysfunction in relapse-onset multiple sclerosis phenotypes: A clinical-imaging evaluation. Multiple sclerosis (Houndmills, Basingstoke, England), 23(4), 577-587. https://doi.org/10.1177/1352458516656809

51. Vitorino, R., Hojjat, S.-P., Cantrell, C. G., Feinstein, A., Zhang, L., Lee, L., O’Connor, P., Carroll, T. J., \& Aviv, R. I. (2016). Regional Frontal Perfusion Deficits in RelapsingRemitting Multiple Sclerosis with Cognitive Decline. American Journal of Neuroradiology, 37(10), 1800-1807. https://doi.org/10.3174/ajnr.A4824

52. Whitfield-Gabrieli, S., \& Nieto-Castanon, A. (2012). Conn: A Functional Connectivity Toolbox for Correlated and Anticorrelated Brain Networks. Brain Connectivity, 2(3), 125-141. https://doi.org/10.1089/brain.2012.0073

53. Xu, J., Wang, J., Fan, L., Li, H., Zhang, W., Hu, Q., \& Jiang, T. (2015). Tractographybased Parcellation of the Human Middle Temporal Gyrus. Scientific Reports, 5. https://doi.org/10.1038/srep18883 


\section{Figure legends}

Figure 1. The whole-brain multivoxel pattern analysis result for relapsing remitting multiple sclerosis and healthy control groups. The significant clusters obtained was at a height threshold of $\mathrm{p}<0.001$ (uncorrected) and FDR-corrected cluster threshold of $\mathrm{p}<0.05$ with the Cluster size (k) at $k=49$. The clusters identified in the figure are: (A) left frontal pole, (B) frontal medial cortex, (C, D) anterior cingulate gyrus, (E, F) right middle frontal gyrus, (G) right middle temporal gyrus, $(\mathrm{H})$ right superior lateral occipital cortex and (I) left posterior middle temporal gyrus.

Figure 2. Post-hoc seed-to-voxel characterization between relapsing remitting multiple sclerosis persons (RRMS) and healthy controls (HC) groups using multivoxel pattern analysis derived clusters (defined by a threshold of $\mathrm{p}<0.001$ (uncorrected) and FDR-corrected cluster threshold of $\mathrm{p}<0.05)$. The regions indicated in blue had decreased resting state functional connectivity (RSFC) and regions in red had increased RSFC in the HC group compared to RRMS. The labelled regions are: (A) left frontal orbital cortex, (B) left amygdala, (C) anterior cingulate gyrus, (D) right paracingulate gyrus, (E) right middle frontal gyrus, (F) right lingual gyrus, (G) left superior lateral occipital cortex, $(\mathrm{H})$ left occipital pole and (I) right superior lateral occipital cortex. 


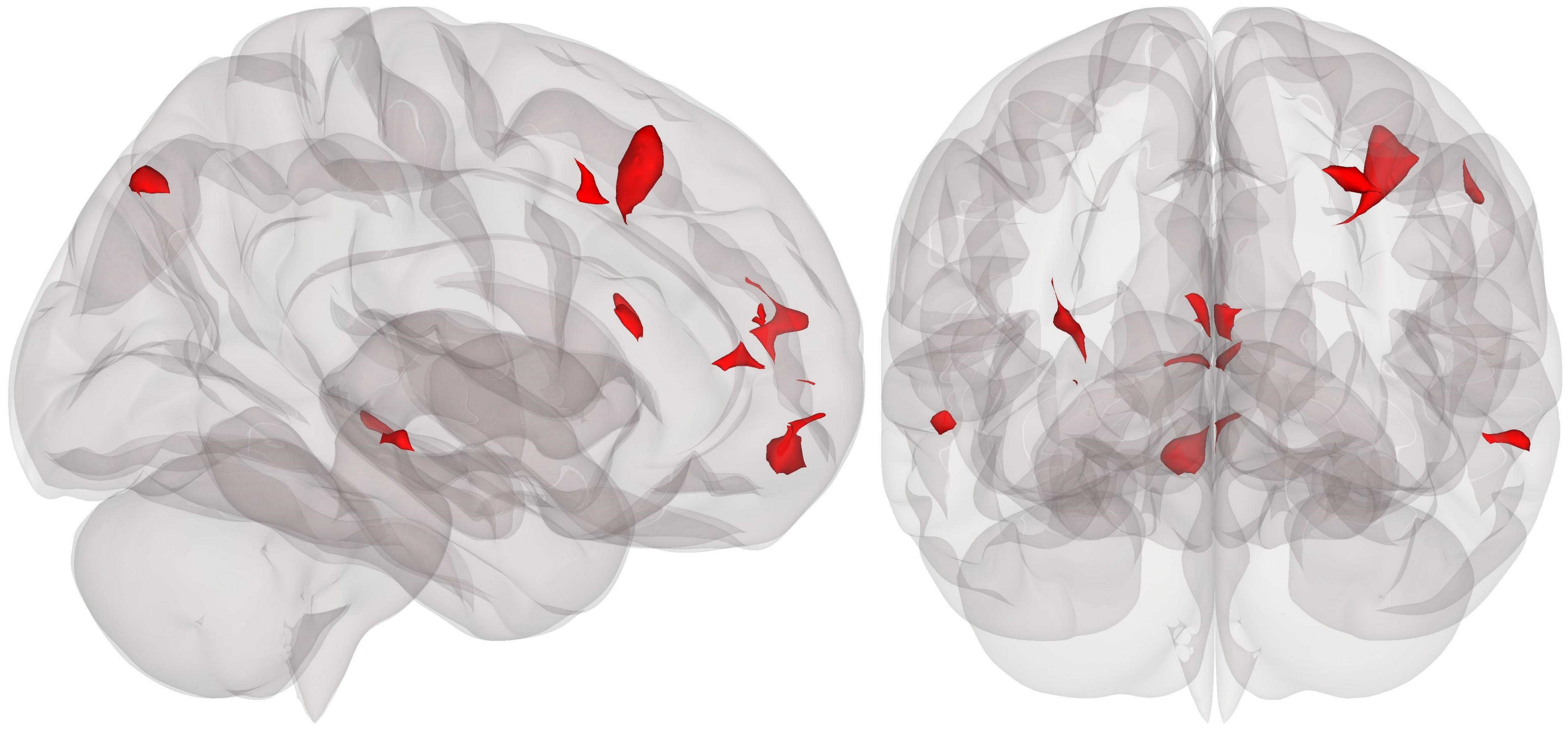

(A)

(1)

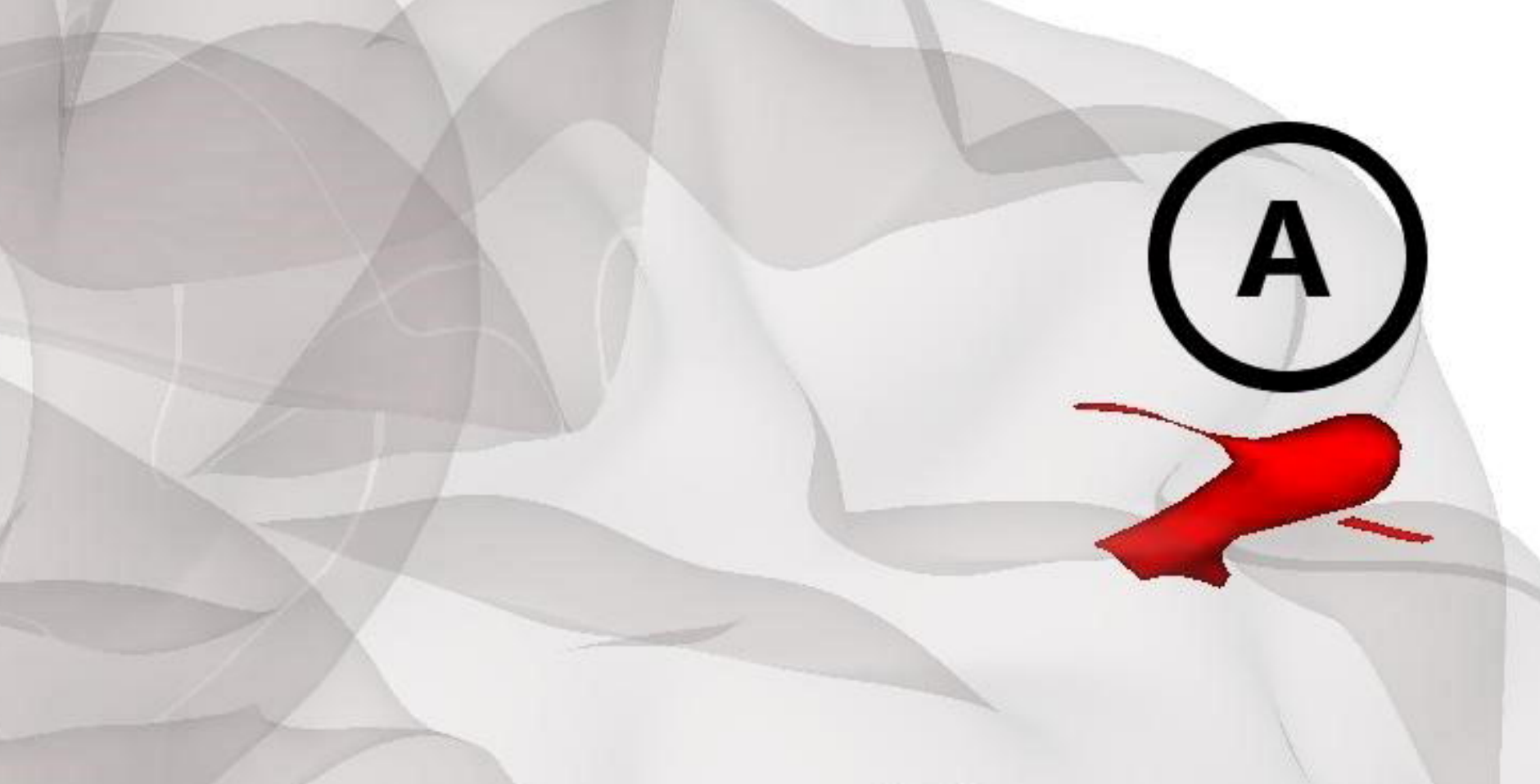

(1) 9 (B)

(1)

(G)

E

(). 
Table 1: Demographic and clinical characteristics of the study participants

\begin{tabular}{|c|c|c|c|}
\hline & $\begin{array}{c}\text { Persons with } \\
\text { RRMS } \\
\mathbf{n}=50\end{array}$ & $\begin{array}{c}\text { Healthy } \\
\text { controls } \\
n=50\end{array}$ & P value \\
\hline \multicolumn{4}{|l|}{ Demographic parameters } \\
\hline Age(years); mean (SD) & $32.80(8.03)$ & $31.96(7.52)$ & 0.591 \\
\hline Females; n (\%) & $32(64)$ & $32(64)$ & - \\
\hline Years of formal education: mean (SD) & $15.16(2.17)$ & $16.1(1.6)$ & 0.015 \\
\hline \multicolumn{4}{|l|}{ Clinical parameters } \\
\hline $\begin{array}{l}\text { Age of symptom onset (years); } \\
\text { mean (SD) }\end{array}$ & $26.78(7.35)$ & - & \\
\hline $\begin{array}{l}\text { Duration of illness (years); } \\
\text { mean (SD) }\end{array}$ & $6.15(4.66)$ & - & \\
\hline Disease modifying therapy; $\mathrm{n}$ (\%) & $43(86)$ & - & \\
\hline EDSS; median (IQR) & $2(1-2.5)$ & - & \\
\hline \multicolumn{4}{|l|}{ Neuropsychology test scores } \\
\hline ACE III-R; mean (SD) & $90.6(6.0)$ & $96.4(2.9)$ & $<0.001$ \\
\hline MMSE; mean (SD) & $29.0(1.6)$ & $29.9(0.303)$ & $<0.001$ \\
\hline
\end{tabular}

Abbreviations: ACE III-R, Addenbrooke's Cognitive Examination III - Revised; EDSS, Expanded Disability Status Scale; HC, Healthy Control; IQR, Interquartile range; MMSE, Mini-mental state examination; n, number; RRMS, Relapsing Remitting Multiple Sclerosis; SD, standard deviation. 
Table 2: Results of post-hoc analysis of the individual clusters derived from group multivoxel pattern analysis between persons with relapsing remitting multiple sclerosis (RRMS) and healthy controls
Cluster
Decreased in RRMS
Increased in RRMS
Cluster 1 Right middle
(HC > RRMS)
(RRMS > HC)
frontal gyrus
Right and left insular cortices
Right and left superior lateral occipital cortices
Right and left frontal poles
Right middle frontal gyrus
Anterior cingulate gyrus

\section{Cluster 2 Frontal medial cortex}

Cluster 3 Left frontal

pole
Right frontal pole

Left middle temporal gyrus (posterior)

Cerebellum Crus 2

Right frontal orbital cortex

Planum temporale

Left inferior frontal gyrus triangularis

Left superior frontal gyrus
Right and left superior lateral occipital cortices

\section{Precuneus}

Right and left frontal poles

\begin{tabular}{ccc}
\hline Cluster 4 & $\begin{array}{c}\text { Anterior } \\
\text { cingulate } \\
\text { gyrus }\end{array}$ & Right superior frontal gyrus \\
& Right and left angular gyri \\
& Right and left posterior middle \\
& temporal gyri \\
& Right frontal pole \\
& Right orbital frontal cortex
\end{tabular}

Cluster 5 Anterior cingulate

gylus

Right posterior supramarginal gyrus

Right middle frontal gyrus

Right frontal pole

Right superior frontal gyrus

Left anterior supramarginal gyrus

Left angular gyrus

Right frontal orbital cortex

Cluster 6 Right lateral

occipital

cortex
Left occipital pole
Left postcentral gyrus

Right superior lateral occipital cortex

\section{$\begin{array}{ll}\text { Cluster } 7 & \text { Right middle }\end{array}$ temporal}

gyrus
Right paracingulate gyrus

\begin{abstract}
Cerebellum
Left occipital pole
Right superior lateral occipital cortex
Precuneus

Right and left superior lateral occipital cortices

Right and left posterior middle temporal gyrus

Right superior frontal gyrus

Right and left superior lateral occipital cortices

Right inferior frontal gyrus pars opercularis
\end{abstract}

Cluster 8 Right middle Right and left anterior supramarginal frontal gyrus

gyri

Right and left frontal poles

Right insular cortex

Left superior frontal gyrus

Anterior cingulate gyrus

Right and left inferior frontal gyri

pars opercularis

Left paracingulate gyrus

Right anterior inferior temporal gyri

Right and left frontal pole

\section{Precuneus}

Right and left frontal poles

Right and left superior lateral occipital cortices Right cerebellum
Cluster 9 Left posterior middle temporal gyrus
Right superior lateral occipital cortices

Left superior parietal lobule

Left cerebellum 6

Left inferior lateral occipital cortex 